[Chem. Pharm. Bull.

36( 3 ) $914-919$ (1988) $]$

\title{
Studies on Sialic Acids. XI. Synthesis of 2-O-Glycosyl Derivatives of $\mathrm{N}$-Acetylneuraminic Acid
}

\author{
Shingo Sato, ${ }^{a}$ Kimio Furuhata, ${ }^{a}$ Masayoshi Itoh, ${ }^{b}$ \\ YOSHIYASU SHITORI, ${ }^{b}$ and HARUO OGURA ${ }^{*, a}$ \\ School of Pharmaceutical Sciences, Kitasato University, ${ }^{a}$ Shirokane, Minato-ku, \\ Tokyo 108, Japan and Central Research Laboratory, MECT Co., Ltd., \\ Nishishinjuku-ku, Tokyo 163, Japan
}

(Received July 27, 1987)

\begin{abstract}
The reactions of methyl $N$-acetyl-4,7,8,9-tetra- $O$-acetyl-2-chloro-2-deoxy- $\beta$-D-neuraminate (2) with $2^{\prime}, 3^{\prime}$-di- $O$-acetylinosine (1) and with $2^{\prime}, 3^{\prime}$-di- $O$-acetyl- $N$-benzoylcytidine $(8)$ under KoenigsKnorr-like reaction conditions gave the corresponding $(2 \rightarrow 5)$ linked disaccharide nucleoside analogues, in yields of $31 \%$ and $23 \%$, respectively. These nucleoside $5^{\prime}-N$-acetylneuraminic acid analogues were converted via saponification or ammonolysis into the final target compounds. The stereochemistry of these compounds was confirmed by analysis.of the proton nuclear magnetic resonance $\left({ }^{1} \mathrm{H}-\mathrm{NMR}\right)$ spectra and measurement of the rate of hydrolysis of the $(2 \rightarrow 5)$ glycosidic linkage.
\end{abstract}

Keywords $-N$-acetylneuraminic acid; Koenigs-Knorr-like reaction; nucleoside 5 '- $N$-acetylneuraminic acid; stereochemistry; ${ }^{1} \mathrm{H}-\mathrm{NMR}$; hydrolysis

We have recently reported ${ }^{1,2,4-6)}$ the syntheses of several 2-O-glycosyl derivatives of $\mathrm{N}$ acetylneuraminic acid and the confirmation of the stereochemistry at the anomeric position of the $N$-acetylneuraminic acid moiety of these compounds by ${ }^{1} \mathrm{H}-\mathrm{NMR}$ spectroscopy, as well as the rate of hydrolysis, and the circular dichroism (CD) spectra. Furthermore, we have reported $^{7,8)}$ that methyl $\left[N\right.$-acetyl-4", $7^{\prime \prime}, 8^{\prime \prime}, 9^{\prime \prime}$-tetra- $O$-acetyl(5-fluoro-2',3'-isopropylideneuridin-5'-yl)- $\alpha$-D-neuraminosid]onate $(13)$ and methyl $\left[N\right.$-acetyl-4", $7^{\prime \prime}, 8^{\prime \prime}, 9^{\prime \prime}$-tetra- $O$-ace$\operatorname{tyl}\left(2^{\prime}, 3^{\prime}\right.$-di-O-acetylinosin-5'-yl)- $\alpha$-D-neuraminosid]onate (3), among the synthesized compounds, induced suppressor $T$ cells and inhibited the activities of sialyltransferases of murine lymphocytes. It was recently reported ${ }^{9)}$ that compound 13 also inhibited experimental pulmonary metastasis of mouse colon adenocarcinoma 26 sublines. Thus, the nucleoside $5^{\prime}$ $\mathrm{N}$-acetylneuraminic acid analogues may show various inhibitory activities in vivo. Therefore, we tried to synthesize these nucleoside $5^{\prime}-N$-acetylneuraminic acid analogues in satisfactory yields.

We wish to report herein the synthesis of $N$-acetyl(inosin-5'-yl)- $\alpha$ - and - $\beta$-D-neuraminosidoic acid (sialosylinosine $\alpha$ - and $\beta$-anomer 6, 7) and $N$-acetyl(cytidin-5'-yl)- $\alpha$ - and $-\beta$-Dneuraminosidoic acid (sialosylcytidine $\alpha$ - and $\beta$-anomer 11, 12), as well as the determination of the configuration at the $\mathrm{C}-2^{\prime \prime}$ position of these compounds by means of the ${ }^{1} \mathrm{H}$-nuclear magnetic resonance $\left({ }^{1} \mathrm{H}-\mathrm{NMR}\right)$ spectral analysis and consideration of the rate of acid hydrolysis.

We examined the Koenigs-Knorr-like reaction, using methyl $\mathrm{N}$-acetyl-4,7,8,9-tetra- $\mathrm{O}$ acetyl-2-chloro-2-deoxy- $\beta$-D-neuraminate (2) as a glycosyl donor, $2^{\prime}, 3^{\prime}$-di- $O$-acetylinosine (1) as a glycosyl acceptor, and silver trifluoromethanesulfonate (AgOTf), which gave the highest yield in the Koenigs-Knorr-like reaction using $2,{ }^{6)}$ or $\mathrm{Hg}$ salt as a catalyst, as shown in Table I. The use of AgOTf as a catalyst and dry $N, N$-dimethylformamide (DMF) as a solvent gave methyl $\left[N\right.$-acetyl-4" ${ }^{\prime \prime}, 7^{\prime \prime}, 8^{\prime \prime}, 9^{\prime \prime}$-tetra- $O$-acetyl( $2^{\prime}, 3^{\prime}$-di- $O$-acetylinosin-5'-yl)- $\alpha$ - and - $\beta$-D-neur- 
aminosid]onate ( 3 and 4 ) in the highest yield. Under the same conditions, the Koenigs-Knorrlike reaction of $2^{\prime}, 3^{\prime}$-di- $O$-acetylcytidine with 2 also gave methyl $\left[N\right.$-acetyl-4" $4^{\prime \prime}, 7^{\prime \prime}, 8^{\prime \prime}, 9^{\prime \prime}$-tetra$O$-acetyl $\left(2^{\prime}, 3^{\prime}\right.$-di- $O$-acetyl- $N$-benzoylcytidin- $5^{\prime}$-yl)- $\alpha$ - and $-\beta$-D-neuraminosid]onate $(9$ and 10 ) in $23 \%$ yield. In addition, methyl $N$-acetyl-4,7,8,9-tetra- $O$-acetyl-2,3-dehydro-2-deoxy-

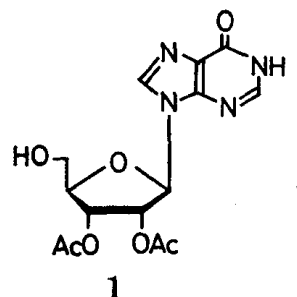

1

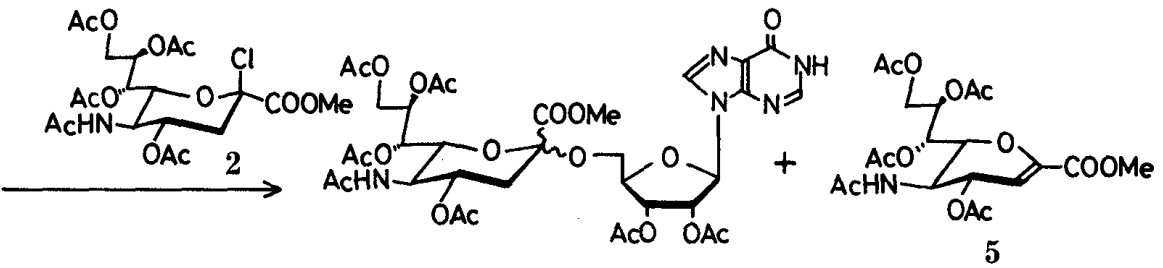

3 : $\alpha$-anomer

4: $\beta$-anomer

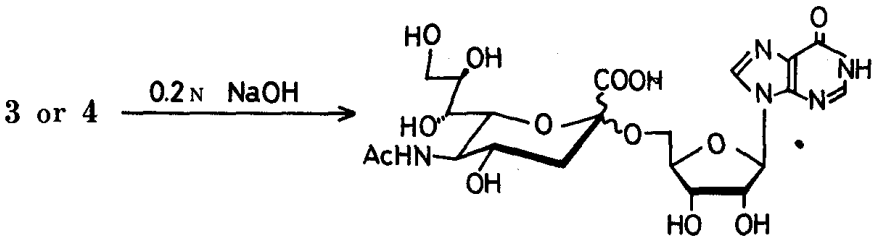

$6: \alpha$-anomer

7 : $\beta$-anomer

Chart 1
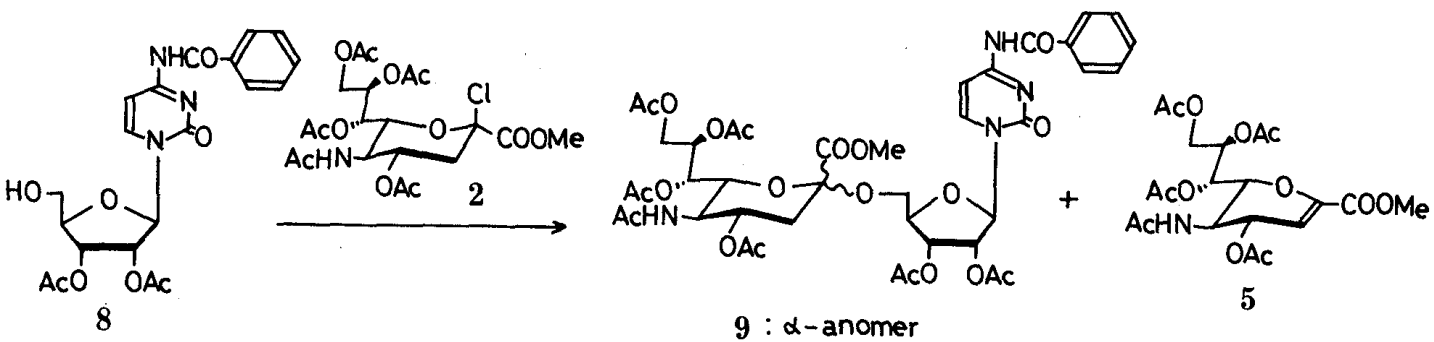

$9: \alpha$-anomer

$10: \beta$-anomer

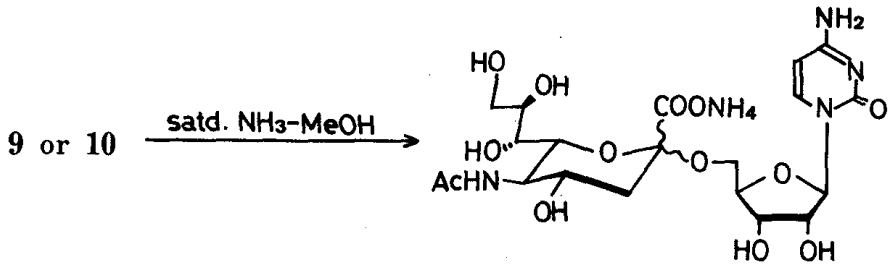

$11: \alpha$-anomer

$12: \beta$-anomer

Chart 2

TABLE I. Koenigs-Knorr-like Reaction of $2^{\prime}, 3^{\prime}$-Di- $O$-Acetylinosine (1) with 2

\begin{tabular}{cllcccc}
\hline \hline Entry & \multicolumn{1}{c}{ Catalyst } & \multicolumn{1}{c}{ Solvent } & $\begin{array}{c}\text { Reaction } \\
\text { temperature }\left({ }^{\circ} \mathrm{C}\right)\end{array}$ & $\begin{array}{c}\text { Reaction } \\
\text { time (d) }\end{array}$ & $\begin{array}{c}\text { Total } \\
\text { yield }(\%)\end{array}$ & $\begin{array}{c}\text { Ratio of } \\
\alpha: \beta\end{array}$ \\
\hline 1 & $\mathrm{Hg}(\mathrm{CN})_{2} / \mathrm{HgBr}_{2}$ & $\mathrm{CH}_{3} \mathrm{NO}_{2}$ or $\mathrm{CH}_{3} \mathrm{CN}$ & $20-25$ & 1 & $5-6$ & $1: 1$ \\
2 & $\mathrm{AgOTf}$ & $\mathrm{THF}_{3}$ & $20-25$ & 1 & 13 & $1: 2$ \\
3 & & $\mathrm{CH}_{2} \mathrm{Cl}_{2}$ & $20-25$ & 1 & 9 & $1: 2$ \\
4 & & DMF & $20-25$ & 1 & 31 & $3: 7$ \\
5 & & & 0 & 2 & 32 & $1: 2$ \\
7 & & & $20-20$ & 2 & 26 & $3: 10$ \\
7 & & & & 1 & 18 & $1: 2$ \\
\hline
\end{tabular}


neuraminate (5) was obtained in $30-50 \%$ yield. The ratios of 3 to 4 and 9 to $\mathbf{1 0}$ were $1: 2$.

The stereochemistry of these compounds was elucidated by consideration of the ${ }^{1} \mathrm{H}$ NMR spectra, the rate of hydrolysis, and the CD spectra.

In the ${ }^{1} \mathrm{H}-\mathrm{NMR}$ spectra, the differences between the chemical shifts at the $\mathrm{H}-3^{\prime \prime}$ (eq) double-doublet resonance of $\alpha$-anomers $(3,6,9$, and 11$)$ and those of $\beta$-anomers $(4,7,10$, and 12) were $+0.17,+0.38,+0.09$, and $+0.34 \mathrm{ppm}$, respectively, as shown in Table II. It is empirically known ${ }^{3,4)}$ that the chemical shift at $\mathrm{H}-3^{\prime \prime}$ (eq) of the $\alpha$-anomer is usually observed at lower field than that of the $\beta$-anomer, and this result followed the empirical rule. In particular, the differences between the chemical shifts at $\mathrm{H}-3^{\prime \prime}$ (eq) of deprotected compounds were larger than those of the protected compounds. This suggests that the $\alpha$-anomer has a greater deshielding effect at $\mathrm{H}-3^{\prime \prime}$ (eq) owing to the carboxyl group, and the effect becomes less when the carboxyl group is esterified. In conclusion, the stereochemistry at the anomeric position could be assessed from the ${ }^{1} \mathrm{H}-\mathrm{NMR}$ data.

Hydrolyses $^{4,5)}$ of two pairs of anomers, 6 and 7, and 11 and 12, were carried out at the concentration of $500 \mu \mathrm{g} / \mathrm{ml}$ in hydrochloric acid $(\mathrm{HCl})$ solution at $60^{\circ} \mathrm{C}$ and the results are shown in Fig. 1. The retention time of $N$-acetylneuraminic acid (Neu5Ac) was $8.8 \mathrm{~min}$ in high performance liquid chromatography (HPLC), as shown in Fig. 2.

In the case of sialosylinosine, the $\alpha$-anomer (6) was decomposed, whereas half of the $\beta$ anomer (7) remained, at $2 \mathrm{~h}$. Sialosylcytidines $(11,12)$ also gave a similar result at $5 \mathrm{~h}$. Thus, the rates of hydrolyses of the $\alpha$-anomers $(\mathbf{6}, \mathbf{1 1})$ were remarkably high in comparison with those of the $\beta$-anomers $(7,12)$. However, $N$-acetylneuraminic acid was decomposed in the acid

TABLE II. ${ }^{1} \mathrm{H}$-NMR Chemical Shifts ( $\delta$ ppm) of H-3" (eq) for Nucleoside 5 '- $N$-Acetylneuraminic Acid Derivatives

\begin{tabular}{ccc|ccc}
\hline \hline Compound & $\mathrm{H}-3^{\prime \prime}(\mathrm{eq})$ & $\Delta(\alpha-\beta)$ & Compound & $\mathrm{H}-3^{\prime \prime}(\mathrm{eq})$ & $\Delta(\alpha-\beta)$ \\
\hline $\mathbf{3}$ & 2.71 & 0.17 & $\mathbf{9}$ & 2.63 & 0.09 \\
$\mathbf{4}$ & 2.54 & & $\mathbf{1 0}$ & 2.54 & \\
$\mathbf{6}$ & 2.66 & 0.38 & $\mathbf{1 1}$ & 2.67 & 0.34 \\
$\mathbf{7}$ & 2.28 & $\mathbf{1 2}$ & 2.33 & \\
\hline
\end{tabular}

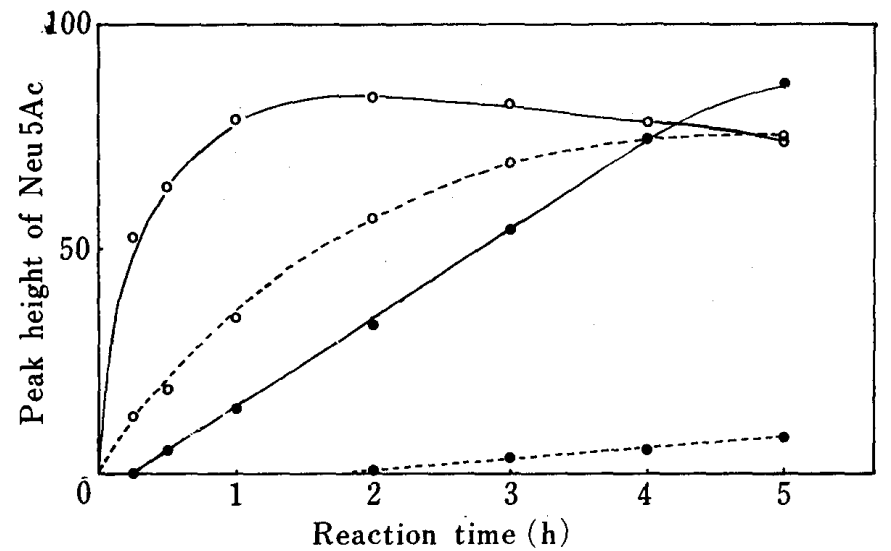

Fig. 1. Acid Hydrolysis of the $(2 \rightarrow 5)$ Glycosidic Linkage Sialosylinosine $\alpha$-Anomer (6) $(-\mathrm{O}-$ ), Sialosylinosine $\beta$-Anomer (7) (---O--), Sialosylcytidine $\alpha$-Anomer (11) (- -), and Sialosylcytidine $\beta$-Anomer (12)

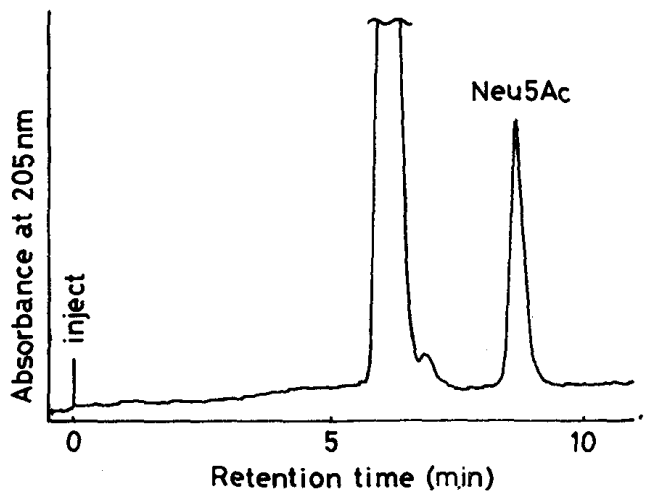

Fig. 2. HPLC Pattern of the 15 min Hydrolyzate of Sialosylinosine $\alpha$-Anomer (6) 


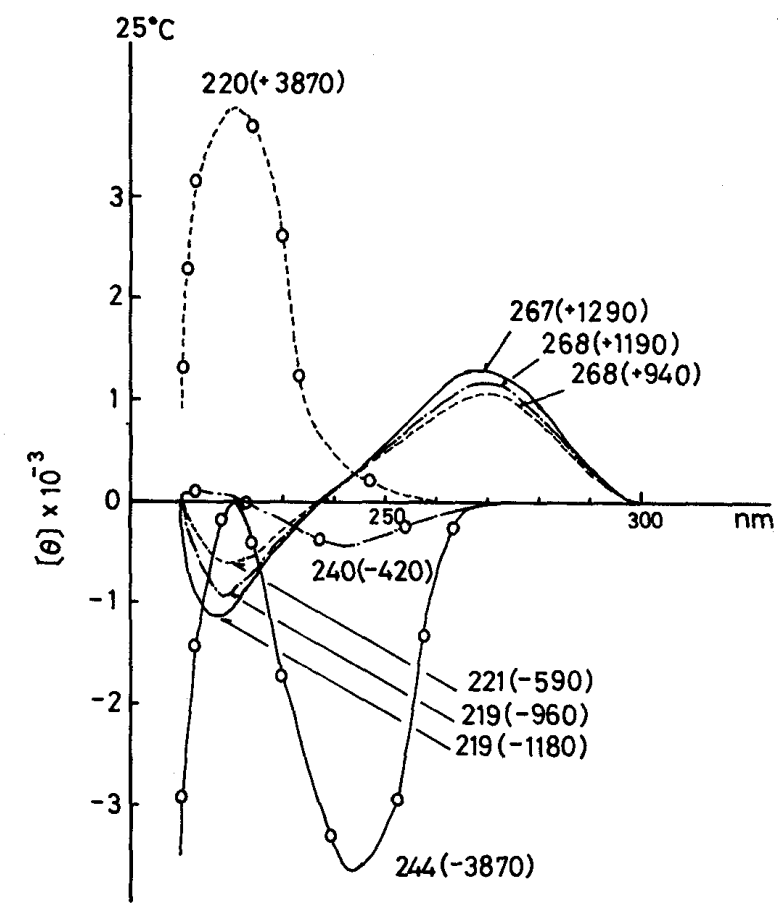

Fig. 3. CD Curves of Sialosylinosine $\alpha$-Anomer (6) (-O-), Sialosylinosine $\beta$-Anomer (7) (--O---), Inosine (-- $\bigcirc-)$, Sialosylcytidine $\alpha$-Anomer (11) (-), Sialosylcytidine $\beta$ Anomer (12) (--.--), and Cytidine (-.-) in Water $(\mathrm{pH}=7)$

solution can be seen from Fig. 1. It is clear that the measurement of the rate of hydrolysis is a useful method for confirmation of anomeric stereochemistry.

Figure 3 shows the CD spectra of sialosylinosines $(6,7)$ and inosine, and sialosylcytidines $(11,12)$ and cytidine. We have already reported ${ }^{1,4-6)}$ the CD spectra of a number of $\mathrm{N}$ acetylneuraminic acid derivatives and the peak around $220 \mathrm{~nm}$ was assigned to the $n-\pi^{*}$ Cotton effect of the carboxyl group. The negative sign of the Cotton effect was assigned to $\alpha$ glycoside and the positive sign to $\beta$-glycoside. ${ }^{1)}$ In sialosylinosines, the $\beta$-anomer (7) showed a positive sign and the $\alpha$-anomer (6) showed a negative sign at around $220 \mathrm{~nm}$. In sialosylcytidines $(11,12)$, the difference of sign at around $220 \mathrm{~nm}$ was not clear. Thus, the difference of the $\mathrm{n}-\pi^{*}$ Cotton effect around $220 \mathrm{~nm}$ due to the carboxyl chromophore did not appear clearly in the nucleoside $5^{\prime}-N$-acetylneuraminic acid derivatives. ${ }^{4)}$

In conclusion, in the nucleoside $5^{\prime}-N$-acetylneuraminic acid derivatives, the stereochemistry at the anomeric position of the $N$-acetylneuraminic acid moiety could be confirmed on the basis of the ${ }^{1} \mathrm{H}-\mathrm{NMR}$ spectra and the rate of acid hydrolysis.

\section{Experimental}

Melting points were measured with a Yamato melting point apparatus and the results are uncorrected. Optical rotations were measured with a JASCO-JIP-4 digital polarimeter. Thin-layer chromatography (TLC) was performed on Silica gel GF254 (Merck) plates, and the spots were detected by ultraviolet (UV) irradiation and with $5 \%$ sulfuric acid solution. Field desorption mass spectra (FD-MS), fast atom bombardment mass spectra (FAB-MS), UV spectra, and infrared (IR) spectra were measured with JEOL JMS-DX300, JEOL JMS-3100, Hitachi 340, and JASCO IR-A2 instruments, respectively. CD spectra were measured in a $0.1 \mathrm{~cm}$ cell with a JASCO J-20 spectrometer. The NMR spectra were measured in chloroform- $d\left(\mathrm{CDCl}_{3}\right)$ with tetramethylsilane (TMS) and in deuterium oxide $\left(\mathrm{D}_{2} \mathrm{O}\right)$ with sodium 3-(trimethylsilyl)-1-propanesulfonate (DSS) as an internal standard, with Varian 90 and Varian 400 spectrometers. Column chromatography was conducted on Silica gel 60 ( $70-230$ mesh, Merck), and gel filtration column chromatography on TOYO PEARL HW-40 fine gel (Toyo Soda).

HPLC - $N$-Acetylneuraminic acid and its derivatives were analyzed by cation exclusion chromatography using an Aminex HPX-87H strong cation exchange resin column $(7.8 \times 300 \mathrm{~mm}$, Bio-Rad Laboratories, Richmond, CA, U.S.A.) at $45^{\circ} \mathrm{C}$. A mobile phase of $0.006 \mathrm{~N}$ sulfuric acid was used at a flow rate of $0.58 \mathrm{ml} / \mathrm{min}$. The column effluent was monitored with a UV detector at $205 \mathrm{~nm}$ (Nihon Seimitsu Kagaku, model NS-310).

Methyl $\left[N\right.$-Acetyl-4", $7^{\prime \prime}, 8^{\prime \prime}, 9^{\prime \prime}$-tetra- $O$-acetyl $\left(2^{\prime}, 3^{\prime}\right.$-di- $O$-acetylinosin-5'-yl)- $\alpha$-and - $\beta$-D-neuraminosid $]$ onates $(3,4)$ 
Compound $2(2.4 \mathrm{mmol})$ and AgOTf $(2.4 \mathrm{mmol})$ were added to a solution of $2^{\prime}, 3^{\prime}$-di-O-acetylinosine (1, $2 \mathrm{mmol})$ and powdered molecular sieves $4 \mathrm{~A}(1.0 \mathrm{~g})$ in dry DMF $(10 \mathrm{ml})$. The mixture was stirred at room temperature overnight in the dark under an argon atmosphere. The reaction mixture was filtered through Celite and washed with dichloromethane $\left(\mathrm{CH}_{2} \mathrm{Cl}_{2}, 50 \mathrm{ml}\right)$. The resulting solution was evaporated in vacuo to a syrup. The residue was extracted with $\mathrm{CH}_{2} \mathrm{Cl}_{2}(30 \mathrm{ml})$ and the $\mathrm{CH}_{2} \mathrm{Cl}_{2}$ extract was washed with a saturated sodium chloride $(\mathrm{NaCl})$ solution $(30 \mathrm{ml} \times 2)$. The $\mathrm{CH}_{2} \mathrm{Cl}_{2}$ extract was dried with anhydrous magnesium sulfate, and evaporated in vacuo. The residue was dissolved in a small volume of chloroform $\left(\mathrm{CHCl}_{3}\right)$, then separated and purified by silica gel column chromatography $\left(\mathrm{CHCl}_{3}-\mathrm{MeOH}, 40: 1-10: 1\right)$ to give the $\alpha$-anomer (3), the $\beta$-anomer (4), and 5 each as an amorphous powder in $153 \mathrm{mg}(9.3 \%), 360 \mathrm{mg}(21.8 \%)$, and $520 \mathrm{mg}$ yields, respectively.

$\alpha$-Anomer (3): $[\alpha]_{\mathrm{D}}^{24}-21.6^{\circ}(c=1.0, \mathrm{MeOH})$. FD-MS $m / z: 826\left(\mathrm{M}^{+}+1\right), 690\left(\mathrm{M}^{+}-135\right), 414\left(\mathrm{M}^{+}-411\right)$. UV $\hat{\lambda}_{\max }^{\mathrm{MeOH}} \mathrm{nm}(\varepsilon): 245(10460)$. IR $v_{\max }^{\text {film }} \mathrm{cm}^{-1}: 3300(\mathrm{NH}), 2950(\mathrm{CH}), 1740$ (ester), 1690 (amide I), 1585 (amide II). Silica gel TLC $\left(\mathrm{CHCl}_{3}-\mathrm{MeOH}, 10: 1\right): R f=0.09 .{ }^{1} \mathrm{H}-\mathrm{NMR}\left(\mathrm{CDCl}_{3}\right) \delta$ : (inosine moiety) $3.662(1 \mathrm{H}, \mathrm{dd}, J=2.3,-11.3 \mathrm{~Hz}$, $\left.\mathrm{H}-5^{\prime} \mathrm{b}\right), 4.101\left(1 \mathrm{H}, \mathrm{dd}, J=2.3,-11.3 \mathrm{~Hz}, \mathrm{H}-5^{\prime} \mathrm{a}\right), 4.405\left(1 \mathrm{H}, \mathrm{dd}, J=2.3,2.5 \mathrm{~Hz}, \mathrm{H}-4^{\prime}\right), 5.661(1 \mathrm{H}, \mathrm{dd}, J=5.0,2.3 \mathrm{~Hz}$, H-3') $5.754\left(1 \mathrm{H}, \mathrm{dd}, J=6.8,5.0 \mathrm{~Hz}, \mathrm{H}-2^{\prime}\right), 6.262\left(1 \mathrm{H}, \mathrm{d}, J=6.8 \mathrm{~Hz}, \mathrm{H}-1^{\prime}\right), 8.139(1 \mathrm{H}, \mathrm{s}, \mathrm{H}-8), 8.225(1 \mathrm{H}, \mathrm{s}, \mathrm{H}-2)$, $12.65(1 \mathrm{H}, \mathrm{s}, 1-\mathrm{NH}) .{ }^{1} \mathrm{H}-\mathrm{NMR}\left(\mathrm{CDCl}_{3}\right) \delta:$ (sialic acid moiety) $1.884(3 \mathrm{H}, \mathrm{s}, \mathrm{NAc}), 2.000(1 \mathrm{H}, \mathrm{dd}, J=9.0,-13.0 \mathrm{~Hz}$, $\left.\mathrm{H}-3^{\prime \prime} \mathrm{ax}\right), 2.018,2.031,2.040,2.138(3 \mathrm{H} \times 4, \mathrm{~s} \times 4, \mathrm{OAc} \times 4), 2.160(6 \mathrm{H}, \mathrm{s} \times 2, \mathrm{OAc} \times 2), 2.712(1 \mathrm{H}, \mathrm{dd}, J=4.6$, $\left.-13.0 \mathrm{~Hz}, \mathrm{H}-3^{\prime \prime} \mathrm{eq}\right), 3.770(3 \mathrm{H}, \mathrm{s}, \mathrm{COOMe}), 4.060\left(1 \mathrm{H}, \mathrm{dd}, J=10.5,10.5 \mathrm{~Hz}, \mathrm{H}-5^{\prime \prime}\right), 4.065(1 \mathrm{H}, \mathrm{dd}, J=5.5$, $\left.-12.5 \mathrm{~Hz}, \mathrm{H}-9^{\prime \prime} \mathrm{b}\right), 4.167\left(1 \mathrm{H}, \mathrm{dd}, J=10.5,2.0 \mathrm{~Hz}, \mathrm{H}-6^{\prime \prime}\right), 4.260\left(1 \mathrm{H}, \mathrm{dd}, J=2.6,-12.5 \mathrm{~Hz}, \mathrm{H}-9^{\prime \prime} \mathrm{a}\right), 4.968(1 \mathrm{H}, \mathrm{m}$, $\left.J=4.6,10.5,9.0 \mathrm{~Hz}, \mathrm{H}-4^{\prime \prime}\right), 5.310\left(1 \mathrm{H}, \mathrm{dd}, J=9.0,2.0 \mathrm{~Hz}, \mathrm{H}-7^{\prime \prime}\right), 5.397(1 \mathrm{H}, \mathrm{m}, \mathrm{NH}), 5.397$ (1H, m, H-8'). Anal. Calcd for $\mathrm{C}_{34} \mathrm{H}_{43} \mathrm{~N}_{5} \mathrm{O}_{19}$ : C, 49.45; H, 5.21; N, 8.48. Found: C, 49.49; H, 5.40; N, 8.57.

$\beta$-Anomer (4): $[\alpha]_{\mathrm{D}}^{24}-5.4^{\circ}(c=1.0, \mathrm{MeOH})$. FD-MS $m / z: 826\left(\mathrm{M}^{+}+1\right), 690\left(\mathrm{M}^{+}-135\right), 414\left(\mathrm{M}^{+}-411\right)$. UV $\lambda_{\max }^{\mathrm{MeOH}} \mathrm{nm}(\varepsilon): 245(8940)$. IR $v_{\max }^{\text {film }} \mathrm{cm}^{-1}: 3250(\mathrm{NH}), 2950(\mathrm{CH}), 1740$ (ester), 1690 (amide I), 1585 (amide II). Silica gel TLC $\left(\mathrm{CHCl}_{3}-\mathrm{MeOH}, 10: 1\right): R f=0.22 .{ }^{1} \mathrm{H}-\mathrm{NMR}\left(\mathrm{CDCl}_{3}\right) \delta$ : (inosine moiety) $3.665(1 \mathrm{H}, \mathrm{dd}, J=2.5,-11.0 \mathrm{~Hz}, \mathrm{H}-$ $5^{\prime}$ b), $3.935\left(1 \mathrm{H}, \mathrm{dd}, J=3.5,-11.0 \mathrm{~Hz}, \mathrm{H}-5^{\prime} \mathrm{a}\right), 4.376\left(1 \mathrm{H}, \mathrm{ddd}, J=4.2,2.5,3.5 \mathrm{~Hz}, \mathrm{H}-4^{\prime}\right), 5.788(1 \mathrm{H}, \mathrm{dd}, J=5.7$, $\left.4.2 \mathrm{~Hz}, \mathrm{H}-3^{\prime}\right), 6.000\left(1 \mathrm{H}, \mathrm{t}, J=5.7 \mathrm{~Hz}, \mathrm{H}-2^{\prime}\right), 6.241\left(1 \mathrm{H}, \mathrm{d}, J=5.7 \mathrm{~Hz}, \mathrm{H}-1^{\prime}\right), 8.190(1 \mathrm{H}, \mathrm{s}, \mathrm{H}-8), 8.370(1 \mathrm{H}, \mathrm{s}, \mathrm{H}-2)$, $12.25(1 \mathrm{H}, \mathrm{s}, 1-\mathrm{NH}) .{ }^{1} \mathrm{H}-\mathrm{NMR}\left(\mathrm{CDCl}_{3}\right) \delta:$ (sialic acid moiety) $1.846\left(1 \mathrm{H}, \mathrm{dd}, J=11.5,-13.0 \mathrm{~Hz}, \mathrm{H}-3^{\prime \prime} \mathrm{ax}\right), 1.977(3 \mathrm{H}$, s, NAc), 1.995, 2.008, 2.030, 2.100, 2.122, $2.165(3 \mathrm{H} \times 6, \mathrm{~s} \times 6, \mathrm{OAc} \times 6), 2.540\left(1 \mathrm{H}, \mathrm{dd}, J=4.8,-13.0 \mathrm{~Hz}, \mathrm{H}-3^{\prime \prime} \mathrm{eq}\right)$, $3.802(3 \mathrm{H}, \mathrm{s}, \mathrm{COOMe}), 3.839\left(1 \mathrm{H}, \mathrm{dd}, J=10.8,2.4 \mathrm{~Hz}, \mathrm{H}-6^{\prime \prime}\right), 3.989\left(1 \mathrm{H}, \mathrm{dd}, J=8.8,-12.2 \mathrm{~Hz}, \mathrm{H}-9^{\prime \prime} \mathrm{b}\right), 4.150(1 \mathrm{H}$, dd, $\left.J=10.3,10.8 \mathrm{~Hz}, \mathrm{H}-5^{\prime \prime}\right), 4.837$ ( $1 \mathrm{H}$, dd, $\left.J=2.8,-12.2 \mathrm{~Hz}, \mathrm{H}-9^{\prime \prime} \mathrm{a}\right), 4.86$ ( $1 \mathrm{H}$, ddd, $J=4.8 \mathrm{~Hz}, 11.5,10.8 \mathrm{~Hz}, \mathrm{H}-$ $\left.4^{\prime \prime}\right), 5.142\left(1 \mathrm{H}\right.$, ddd, $\left.J=2.8,8.8,2.4 \mathrm{~Hz}, \mathrm{H}-8^{\prime \prime}\right), 5.247\left(1 \mathrm{H}, \mathrm{t}, J=2.4 \mathrm{~Hz}, \mathrm{H}^{-} 7^{\prime \prime}\right), 6.184(1 \mathrm{H}, \mathrm{d}, J=10.3 \mathrm{~Hz}, \mathrm{NH})$. Anal. Calcd for $\mathrm{C}_{34} \mathrm{H}_{43} \mathrm{~N}_{5} \mathrm{O}_{19}: \mathrm{C}, 49.45 ; \mathrm{H}, 5.21 ; \mathrm{N}, 8.48$. Found: $\mathrm{C}, 49.46 ; \mathrm{H}, 5.26 ; \mathrm{N}, 8.41$.

$\boldsymbol{N}$-Acetyl(inosin-5'-yl)- $\alpha$ - and $-\boldsymbol{\beta}$-D-neuraminosidoic Acids $(6,7)-$ - $2 \mathrm{~N} \mathrm{NaOH}$ solution $(2 \mathrm{ml})$ was added to a solution of 3 or 4 in $\mathrm{H}_{2} \mathrm{O}(10 \mathrm{ml})$ and the mixture was stirred for $2 \mathrm{~h}$ at room temperature. Dowex $50 \mathrm{~W} \times 8\left(\mathrm{H}^{+}\right)$resin was added to the reaction mixture, which was adjusted to $\mathrm{pH} 3-4$ in an ice bath. The whole was filtered and washed with water. The resulting solution was freeze-dried to yield $40 \mathrm{mg}(82 \%)$ of the $\alpha$-anomer $(6)$, or $48 \mathrm{mg}(98 \%)$ of the $\beta$ anomer (7), as white powder.

$\alpha$-Anomer $(6):[\alpha]_{\mathrm{D}}^{25}-21.4^{\circ}\left(c=1.0, \mathrm{H}_{2} \mathrm{O}, \mathrm{pH}=7\right)$. FD-MS $m / z: 559\left(\mathrm{M}^{+}\right), 423\left(\mathrm{M}^{+}-136\right), 291\left(\mathrm{M}^{+}-268\right), 136$ $\left(\mathrm{M}^{+}-423\right)$. UV $\lambda_{\max }^{\mathrm{H}_{2} \mathrm{O}(\mathrm{pH}=7)} \mathrm{nm}(\varepsilon): 248(9170) . \mathrm{IR} v_{\max }^{\mathrm{film}} \mathrm{cm}^{-1}: 3350(\mathrm{NH}), 1690$ (amide I), 1580 (amide II). ${ }^{1} \mathrm{H}-\mathrm{NMR}$ $\left(\mathrm{D}_{2} \mathrm{O}\right) \delta: 1.94(3 \mathrm{H}, \mathrm{s}, \mathrm{NAc}), 2.66\left(1 \mathrm{H}, \mathrm{dd}, J=5.0,-12.0 \mathrm{~Hz}, \mathrm{H}-3^{\prime \prime} \mathrm{eq}\right), 6.02\left(1 \mathrm{H}, \mathrm{d}, J=4.2 \mathrm{~Hz}, \mathrm{H}-1^{\prime}\right), 8.12(1 \mathrm{H}, \mathrm{s}, 2-$ $\mathrm{H}), 8.33(1 \mathrm{H}, \mathrm{s}, 8-\mathrm{H}) .{ }^{13} \mathrm{C}-\mathrm{NMR}\left(\mathrm{D}_{2} \mathrm{O}\right) \delta: 91.10\left(\mathrm{C}-1^{\prime}\right), 103.14\left(\mathrm{C}-2^{\prime \prime}\right), 176.22,177.96\left(1^{\prime \prime}-\mathrm{COOH}, 4^{\prime \prime}-\mathrm{NAc}\right)$. Anal. Calcd for $\mathrm{C}_{21} \mathrm{H}_{29} \mathrm{~N}_{5} \mathrm{O}_{13} \cdot 2.5 \mathrm{H}_{2} \mathrm{O}: \mathrm{C}, 41.72 ; \mathrm{H}, 5.63 ; \mathrm{N}, 11.59$. Found: $\mathrm{C}, 41.85 ; \mathrm{H}, 5.69 ; \mathrm{N}, 11.47$.

$\beta$-Anomer (7): $[\alpha]_{\mathrm{D}}^{25}-10.2^{\circ}\left(c=1.0, \mathrm{H}_{2} \mathrm{O}, \mathrm{pH}=7\right)$. FD-MS $m / z: 559\left(\mathrm{M}^{+}\right), 423\left(\mathrm{M}^{+}-136\right), 291\left(\mathrm{M}^{+}-268\right), 136$ $\left(\mathrm{M}^{+}-423\right)$. UV $\lambda_{\max }^{\mathrm{H}_{2} \mathrm{O}(\mathrm{pH}=7)} \mathrm{nm}(\varepsilon): 248(11970)$. IR $v_{\max }^{\mathrm{film}} \mathrm{cm}^{-1}: 3300(\mathrm{NH}), \mathrm{I} 685$ (amide I), 1610 (amide II). ${ }^{1} \mathrm{H}-\mathrm{NMR}$ $\left(\mathrm{D}_{2} \mathrm{O}\right) \delta: 1.91(3 \mathrm{H}, \mathrm{s}, \mathrm{NAc}), 2.28\left(1 \mathrm{H}, \mathrm{dd}, J=4.3,-13.5 \mathrm{~Hz}, \mathrm{H}-3^{\prime \prime} \mathrm{eq}\right), 5.97\left(1 \mathrm{H}, \mathrm{d}, J=4.2 \mathrm{~Hz}, \mathrm{H}-1^{\prime}\right), 8.08(1 \mathrm{H}, \mathrm{s}, \mathrm{H}-$ 2), $8.25(1 \mathrm{H}, \mathrm{s}, \mathrm{H}-8) .{ }^{13} \mathrm{C}-\mathrm{NMR}\left(\mathrm{D}_{2} \mathrm{O}\right) \delta: 91.02\left(\mathrm{C}-1^{\prime}\right), 103.31$ (C-2'), 177.73, 177.83 (4"'-NAc, 1"'-COOH). Anal. Calcd for $\mathrm{C}_{21} \mathrm{H}_{29} \mathrm{~N}_{5} \mathrm{O}_{13} \cdot 2.5 \mathrm{H}_{2} \mathrm{O}: \mathrm{C}, 41.72 ; \mathrm{H}, 5.63 ; \mathrm{N}, 11.59$. Found: $\mathrm{C}, 41.45 ; \mathrm{H}, 5.34 ; \mathrm{N}, 11.46$.

Methyl [ $N$-Acetyl-4" $7^{\prime \prime}, 8^{\prime \prime}, 9^{\prime \prime}$-tetra- $O$-acetyl $\left(2^{\prime}, 3^{\prime}\right.$-di- $O$-acetyl- $N$-benzoylcytidin-5'-yl)- $\alpha$ - and - $\beta$-D-neuraminosid]onates $(9,10)$ Compound $2(12 \mathrm{mmol})$ and $\operatorname{AgOTf}(12 \mathrm{mmol})$ were added to a solution of $8(10 \mathrm{mmol})$ and powdered molecular sieves $4 \mathrm{~A}(3.0 \mathrm{~g})$ in dry DMF $(40 \mathrm{ml})$. The mixture was stirred at room temperature overnight in the dark under an argon atmosphere. The reaction mixture was filtered through Celite and washed with $\mathrm{CH}_{2} \mathrm{Cl}_{2}$ $(200 \mathrm{ml})$. The resulting solution was evaporated to a syrup in vacuo. The residue was extracted with $\mathrm{CH}_{2} \mathrm{Cl}_{2}(150 \mathrm{ml})$. The $\mathrm{CH}_{2} \mathrm{Cl}_{2}$ extract was washed with a saturated $\mathrm{NaCl}$ solution $(50 \mathrm{ml} \times 2)$, dried with anhydrous magnesium sulfate, and evaporated in vacuo. The residue was separated by silica gel column chromatography $\left(\mathrm{CHCl}_{3}-\mathrm{MeOH}_{2} 20: 1\right)$. The eluate was separated and purified by silica gel column chromatography (benzene-acetone, $2: 1$ ), affording $0.68 \mathrm{~g}$ $(7.5 \%)$ of 9 and $1.22 \mathrm{~g}(13.5 \%)$ of 10 , each as a white powder. 9 was recrystallized from ethyl acetate as colorless prisms. Furthermore, 5 was collected in $2.80 \mathrm{~g}$ yield.

$\alpha$-Anomer (9): $\operatorname{mp} 207.5-209^{\circ} \mathrm{C} .[\alpha]_{\mathrm{D}}^{24}+24.1^{\circ}\left(c=1.08, \mathrm{CHCl}_{3}\right)$. FD-MS $m / z: 905\left(\mathrm{M}^{+}-1\right) . \operatorname{IR} v_{\max }^{\mathrm{film}} \mathrm{cm}^{-1}$ : 3270 (NH), 1740 (ester), 1650 (amide I), 1550 (amide II). Silica gel TLC (benzene-acetone, $1: 1$ ), $R f=0.23 .{ }^{1} \mathrm{H}-\mathrm{NMR}$ $\left(\mathrm{CDCl}_{3}\right) \delta$ : (cytidine moiety) $3.627\left(1 \mathrm{H}, \mathrm{dd}, J=2.0,-11.2 \mathrm{~Hz}, \mathrm{H}-5^{\prime \prime} \mathrm{b}\right), 4.145\left(1 \mathrm{H}, \mathrm{dd}, J=2,0,-11.2 \mathrm{~Hz}, \mathrm{H}-5^{\prime} \mathrm{a}\right)$, $4.377\left(1 \mathrm{H}, \mathrm{dd}, J=2.8,2.0 \mathrm{~Hz}, \mathrm{H}-4^{\prime}\right), 5.395\left(1 \mathrm{H}, J=6.5,5.0 \mathrm{H}, \mathrm{H}-2^{\prime}\right), 5.522\left(1 \mathrm{H}, \mathrm{dd}, J=5.0,2.8 \mathrm{~Hz}, \mathrm{H}-3^{\prime}\right), 6.410(1 \mathrm{H}$, 
d, $\left.J=6.5 \mathrm{~Hz}, \mathrm{H}-\mathrm{I}^{\prime}\right), 7.52(3 \mathrm{H}, \mathrm{m}, \mathrm{Ph}), 7.62(2 \mathrm{H}, \mathrm{m}, \mathrm{Ph}), 7.89(1 \mathrm{H}, \mathrm{d}, J=7.6 \mathrm{~Hz}, \mathrm{H}-5), 8.22(1 \mathrm{H}, \mathrm{d}, J=7.6 \mathrm{~Hz}, \mathrm{H}-6)$ ${ }^{1} \mathrm{H}$-NMR $\left(\mathrm{CDCl}_{3}\right) \delta:$ (sialic acid moiety) $1.890(3 \mathrm{H}, \mathrm{s}, \mathrm{NAc}), 2.036,2.056,2.072,2.136,2.152,2.162(18 \mathrm{H}, \mathrm{s} \times 6$, $\mathrm{OAc} \times 6), 2.63\left(1 \mathrm{H}, \mathrm{dd}, J=4.8,-12.5 \mathrm{~Hz}, \mathrm{H}-3^{\prime \prime} \mathrm{eq}\right), 3.84(3 \mathrm{H}, \mathrm{s}, \mathrm{COOMe}), 4.064\left(1 \mathrm{H}, \mathrm{dd}, J=4.5,-12.2 \mathrm{~Hz}, \mathrm{H}-9^{\prime \prime} \mathrm{b}\right)$, $4.065\left(1 \mathrm{H}, \mathrm{dd}, J=10.5,10.5 \mathrm{~Hz}, \mathrm{H}-5^{\prime \prime}\right), 4.09\left(1 \mathrm{H}, \mathrm{dd}, J=10.5,2.2 \mathrm{~Hz}, \mathrm{H}-6^{\prime \prime}\right), 4.260(1 \mathrm{H}, \mathrm{dd}, J=2.6,-12.2 \mathrm{~Hz}, \mathrm{H}-$ $9^{\prime \prime}$ a), $5.005\left(1 \mathrm{H}, \mathrm{ddd}, J=4.8,12.0,10.5 \mathrm{~Hz}, \mathrm{H}-4^{\prime \prime}\right), 5.32 .7\left(1 \mathrm{H}, \mathrm{dd}, J=9.0,2.2 \mathrm{~Hz}, \mathrm{H}-7^{\prime \prime}\right), 5.395(1 \mathrm{H}, \mathrm{ddd}, J=2.6,4.5$, $9.0 \mathrm{~Hz}, \mathrm{H}-8), 8.22\left(1 \mathrm{H}, \mathrm{d}, J=10.5 \mathrm{~Hz}, 5^{\prime \prime}-\mathrm{NH}\right)$. Anal. Calcd for $\mathrm{C}_{40} \mathrm{H}_{48} \mathrm{~N}_{4} \mathrm{O}_{20}: \mathrm{C}, 53.10 ; \mathrm{H}, 5.31 ; \mathrm{N}, 6.19$. Found: C, $53.26 ; \mathrm{H}, 5.28 ; \mathrm{N}, 5.85$.

$\beta$-Anomer (10): $[\alpha]_{\mathrm{D}}^{24}+25.4^{\circ}\left(c=0.93, \mathrm{CHCl}_{3}\right)$. FD-MS $m / z: 905\left(\mathrm{M}^{+}+1\right) . \mathrm{IR} v_{\max }^{\mathrm{film}} \mathrm{cm}^{-1}: 3280(\mathrm{NH}), 1740$ (ester), 1660 (amide I), 1550 (amide II). Silica gel TLC (benzene-acetone, 1:1), $R f=0.29 .{ }^{1} \mathrm{H}-\mathrm{NMR}\left(\mathrm{CDCl}_{3}\right) \delta$ : (cytidine moiety) $3.732\left(1 \mathrm{H}, \mathrm{dd}, J=2.5,-11.8 \mathrm{~Hz}, \mathrm{H}-5^{\prime} \mathrm{b}\right), 4.167\left(1 \mathrm{H}, \mathrm{dd}, J=1.9,-11.8 \mathrm{~Hz}, \mathrm{H}-5^{\prime} \mathrm{a}\right), 4.263$ (1 H, ddd, $\left.J=6.5,2.5,1.9 \mathrm{~Hz}, \mathrm{H}-4^{\prime}\right), 5.57\left(1 \mathrm{H}, \mathrm{dd}, J=3.0,6.5 \mathrm{~Hz}, \mathrm{H}-2^{\prime}\right), 5.83\left(1 \mathrm{H}, \mathrm{d}, J=3.0 \mathrm{~Hz}, \mathrm{H}-1^{\prime}\right), 5.597(1 \mathrm{H}, \mathrm{t}, J=6.5 \mathrm{~Hz}$, H-3') $7.43(1 \mathrm{H}, \mathrm{d}, J=7.5 \mathrm{~Hz}, \mathrm{H}-5), 7.43(1 \mathrm{H}, \mathrm{m}, \mathrm{Ph}), 7.54(2 \mathrm{H}, \mathrm{m}, \mathrm{Ph}), 7.85(1 \mathrm{H}, \mathrm{d}, J=7.5 \mathrm{~Hz}, \mathrm{H}-6), 7.85(2 \mathrm{H}, \mathrm{m}$, $\mathrm{Ph}) .{ }^{1} \mathrm{H}-\mathrm{NMR}\left(\mathrm{CDCl}_{3}\right) \delta:$ (sialic acid moiety) $1.891\left(1 \mathrm{H}, \mathrm{dd}, J=11.0,-13.0 \mathrm{~Hz}, \mathrm{H}-3{ }^{\prime \prime} \mathrm{ax}\right), 1.95(3 \mathrm{H}, \mathrm{s}, \mathrm{NAc}), 1.959$, $1.998,2.048,2.141(12 \mathrm{H}, \mathrm{s} \times 4, \mathrm{OAc} \times 4), 2.113 \times 2(6 \mathrm{H}, \mathrm{s}, \mathrm{OAc} \times 2), 2.541\left(1 \mathrm{H}, \mathrm{dd}, J=5.0,-13.0 \mathrm{~Hz}, \mathrm{H}-3^{\prime \prime} \mathrm{eq}\right), 3.80$ $(3 \mathrm{H}, \mathrm{s}, \mathrm{COOMe}), 4.002\left(1 \mathrm{H}, \mathrm{dd}, J=9.0,-12.0 \mathrm{~Hz}, \mathrm{H}-9^{\prime \prime} \mathrm{b}\right), 4.167\left(1 \mathrm{H}, \mathrm{dd}, J=10.5 \mathrm{~Hz}, \mathrm{H}-5^{\prime \prime}\right), 4.472(1 \mathrm{H}, \mathrm{dd}, J=$ $\left.10.5,2.0 \mathrm{~Hz}, \mathrm{H}-6^{\prime \prime}\right), 4.992\left(1 \mathrm{H}, \mathrm{dd}, J=2.2,-12.0 \mathrm{~Hz}, \mathrm{H}-9^{\prime \prime} \mathrm{a}\right), 5.32\left(1 \mathrm{H}, \mathrm{m}, \mathrm{H}-8^{\prime \prime}\right), 5.355\left(1 \mathrm{H}, \mathrm{m}, \mathrm{H}-7^{\prime \prime}\right), 7.14(1 \mathrm{H}, \mathrm{d}$, $\left.J=10.5 \mathrm{~Hz}, 5^{\prime \prime}-\mathrm{NH}\right)$. Anal. Calcd for $\mathrm{C}_{40} \mathrm{H}_{48} \mathrm{~N}_{4} \mathrm{O}_{20}: \mathrm{C}, 53.10 ; \mathrm{H}, 5.31 ; \mathrm{N}, 6.19$. Found: C, 53.58; H, 5.24;, 6.15 .

Ammonium [ $\boldsymbol{N}$-Acetyl(cytidin-5'-yl)- $\alpha$ - and $-\beta$-D-neuraminosid]onate $(11,12)$ _- Saturated ammonia gas was passed through a solution of $\mathbf{9}$, or $10(0.066 \mathrm{mmol})$ in methanol $(15 \mathrm{ml})$ and the reaction mixture was left in a refrigerator overnight. It was evaporated in vacuo to remove methanol and purified by gel filtration column chromatography $(2.2 \times 30 \mathrm{~cm}$; mobile phase, methanol). The eluate was evaporated in vacuo to give $11(35 \mathrm{mg}, 98 \%)$, or $12(35 \mathrm{mg}, 98 \%)$, each as an amorphous solid.

$\alpha$-Anomer (11): $[\alpha]_{\mathrm{D}}^{23}+15.1^{\circ}\left(c=0.97, \mathrm{H}_{2} \mathrm{O}, \mathrm{pH}=7\right)$. FAB-MS (Xe, glycerol) $m / z: 534\left(\mathrm{M}^{+}-\mathrm{NH}_{3}\right), 291$ $\left(\mathrm{M}^{+}-260\right), 244\left(\mathrm{M}^{+}-307\right)$. UV $\lambda_{\max }^{\mathrm{H}_{2} \mathrm{O}(\mathrm{pH}=7)} \mathrm{nm}(\varepsilon): 269(7200)$. IR $v_{\max }^{\mathrm{KBr}} \mathrm{cm}^{-1}: 3350(\mathrm{NH}), 1660$ (amide I), 1490 (amide II). ${ }^{1} \mathrm{H}-\mathrm{NMR}\left(\mathrm{D}_{2} \mathrm{O}\right) \delta:$ (cytidine moiety) $4.178\left(1 \mathrm{H}, \mathrm{dd}, J=3.7,5.0 \mathrm{~Hz}, \mathrm{H}-2^{\prime}\right), 4.221\left(1 \mathrm{H}, \mathrm{t}, J=5.0 \mathrm{~Hz}, \mathrm{H}-3^{\prime}\right)$, $5.806\left(1 \mathrm{H}, \mathrm{d}, J=3.7 \mathrm{~Hz}, \mathrm{H}^{\prime} \mathrm{l}^{\prime}\right), 5.969(1 \mathrm{H}, \mathrm{d}, J=7.3 \mathrm{~Hz}, \mathrm{H}-6), 7.801(1 \mathrm{H}, \mathrm{d}, J=7.3 \mathrm{~Hz}, \mathrm{H}-5)$. (sialic acid moiety) $1.753\left(1 \mathrm{H}, \mathrm{dd}, J=11.6,-12.5 \mathrm{~Hz}, \mathrm{H}-3^{\prime \prime} \mathrm{ax}\right), 1.900$ (3H, s, NHAc), 2.670 (1H, dd, $\left.J=4.5,-12.5 \mathrm{~Hz}, \mathrm{H}-3^{\prime \prime} \mathrm{eq}\right), 3.665$ $\left(1 \mathrm{H}, \mathrm{ddd}, J=4.5,11.6,10.3 \mathrm{~Hz}, \mathrm{H}-4^{\prime \prime}\right), 3.83\left(1 \mathrm{H}, \mathrm{t}, J=10.3 \mathrm{~Hz}, \mathrm{H}-5^{\prime}\right), 3.88\left(1 \mathrm{H}, \mathrm{dd}, J=3.4,-11.0 \mathrm{~Hz}, \mathrm{H}-9^{\prime \prime} \mathrm{b}\right), 3.915$ $\left(1 \mathrm{H}, \mathrm{dd}, J=3.1,-11.0 \mathrm{~Hz}, \mathrm{H}-9^{\prime \prime} \mathrm{a}\right), 4.144\left(1 \mathrm{H}, \mathrm{m}, \mathrm{H}-8^{\prime \prime}\right) .{ }^{13} \mathrm{C}-\mathrm{NMR}\left(\mathrm{D}_{2} \mathrm{O}\right) \delta: 37.80\left(\mathrm{C}-3^{\prime \prime}\right), 62.42\left(\mathrm{C}-5^{\prime}\right), 63.87(\mathrm{C}-$ $\left.9^{\prime \prime}\right), 81.57\left(\mathrm{C}-9^{\prime \prime}\right), 98.35\left(\mathrm{C}-2^{\prime \prime}\right)$. Anal. Calcd for $\mathrm{C}_{30} \mathrm{H}_{29} \mathrm{~N}_{4} \mathrm{O}_{13} \cdot \mathrm{NH}_{4}: \mathrm{C}, 43.56 ; \mathrm{H}, 5.99 ; \mathrm{N}, 12.70$. Found: C, $43.87 ; \mathrm{H}$, $6.46 ; \mathrm{N}, 14.31$.

$\beta$-Anomer $(12):[\alpha]_{\mathrm{D}}^{23}+15.6\left(c=1.03, \mathrm{H}_{2} \mathrm{O}, \mathrm{pH}=7\right)$. FAB-MS (Xe, glycerol) $m / z: 534\left(\mathrm{M}^{+}-\mathrm{NH}_{3}\right), 291$ $\left(\mathrm{M}^{+}-260\right), 244\left(\mathrm{M}^{+}-307\right)$. UV $\lambda_{\max }^{\mathrm{H}_{2} \mathrm{O}(\mathrm{pH}=7)} \mathrm{nm}(\varepsilon): 269(7800) . \mathrm{IR} v_{\max }^{\mathrm{KBr}} \mathrm{cm}^{-1}: 3300(\mathrm{NH}), 1650$ (amide I), 1490 (amide II). ${ }^{1} \mathrm{H}-\mathrm{NMR}\left(\mathrm{D}_{2} \mathrm{O}\right) \delta:$ (cytidine moiety) $3.411(1 \mathrm{H}, \mathrm{d}, J=5.0,-12.0 \mathrm{~Hz}, \mathrm{H}-5$ 'a $), 4.176(1 \mathrm{H}, \mathrm{t}, J=5.0 \mathrm{~Hz}, \mathrm{H}-$ $\left.3^{\prime}\right), 4.212\left(1 \mathrm{H}, \mathrm{dd}, J=3.4,5.0 \mathrm{~Hz}, \mathrm{H}-2^{\prime}\right), 5.787\left(1 \mathrm{H}, \mathrm{d}, J=3.4 \mathrm{~Hz}, \mathrm{H}-1^{\prime}\right), 5.957(1 \mathrm{H}, \mathrm{d}, J=7.5 \mathrm{~Hz}, \mathrm{H}-6), 7.757$ (1 H, d, $J=7.5 \mathrm{~Hz}, \mathrm{H}-5) .{ }^{1} \mathrm{H}-\mathrm{NMR}\left(\mathrm{D}_{2} \mathrm{O}\right) \delta:$ (sialic acid moiety) $1.608\left(1 \mathrm{H}, \mathrm{dd}, J=11.2,-13.0 \mathrm{~Hz}, \mathrm{H}-3^{\prime \prime} \mathrm{ax}\right), 1.950(3 \mathrm{H}, \mathrm{s}$, NHAc), $2.332\left(1 \mathrm{H}, \mathrm{dd}, J=5.0,-13.0 \mathrm{~Hz}, \mathrm{H}-3^{\prime \prime}\right.$ eq) $, 3.547\left(1 \mathrm{H}, \mathrm{dd}, J=6.0,-12.0 \mathrm{~Hz}, \mathrm{H}-9^{\prime \prime} \mathrm{b}\right), 3.712(1 \mathrm{H}, \mathrm{dd}, J=3.0$, $\left.-12.0 \mathrm{~Hz}, \mathrm{H}-9^{\prime \prime} \mathrm{a}\right), 3.937$ (1 H, m, H-4"'), 4.115 (1H, m, H-8 $\left.{ }^{\prime \prime}\right) .{ }^{13} \mathrm{C}-\mathrm{NMR}\left(\mathrm{D}_{2} \mathrm{O}\right) \delta: 36.92\left(\mathrm{C}-3^{\prime \prime}\right), 61.39\left(\mathrm{C}-5^{\prime}\right), 62.82$

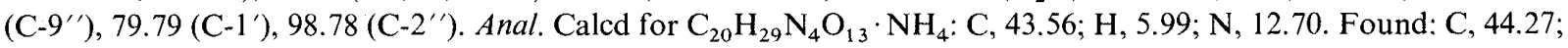
$\mathrm{H}, 6.13 ; \mathrm{N}, 12.42$.

Acknowledgement This work was supported in part by Grants-in-Aid for Scientific Research (59870071) and for Cancer Research (61010096) from the Ministry of Education, Science and Culture of Japan, and a Grant-in-Aid for Scientific Research (Project-1) from the School of Pharmaceutical Sciences, Kitasato University, Japan.

\section{References}

1) H. Ogura and K. Furuhata, Tetrahedron Lett., 22, 4265 (1981).

2) H. Ogura and K. Furuhata, Yuki Gosei Kagaku Kyokaishi, 42, 536 (1984).

3) R. Schauer, "Sialic Acids, Chemistry, Metabolism, and Function," Springer-Verlag, Vienna, New York, 1982, pp. $128-172$.

4) H. Ogura, K. Furuhata, M. Itoh, and Y. Shitori, Carbohydr. Res., 158, 37 (1986).

5) K. Furuhata, K. Anazawa, M. Itoh, Y. Shitori, and H. Ogura, Chem. Pharm. Bull., 34, 2725 (1986).

6) S. Sato, S. Fujita, K. Furuhata, H. Ogura, S. Yoshimura, M. Itoh, and Y. Shitori, Chem. Pharm. Bull., 35, 4043 (1987).

7) I. Kijima, K. Ezawa, S. Toyoshima, K. Furuhata, H. Ogura, and T. Osawa, Chem. Pharm. Bull., 30, 3278 (1982)

8) I. Kijima-Suda, S. Toyoshima, M. Itoh, K. Furuhata, H. Ogura, and T. Osawa, Chem. Pharm. Bull., 33, 730 (1985).

9) I. Kijima-Suda, Y. Miyamoto, S. Toyoshima, M. Itoh, and T. Osawa, Cancer Res., 46, 858 (1986). 